\title{
Extending Darwin Core to incorporate data about material condition and absolute deep time
}

\author{
Laura Brenskelle ${ }^{\ddagger}$, John Wieczorek ${ }^{\S}$, Robert Guralnick ${ }^{\ddagger}$, Kitty Emery $^{\ddagger}$, Michelle LeFebvre ${ }^{\ddagger}$ \\ ‡ Florida Museum of Natural History, University of Florida, Gainesville, United States of America \\ $\S$ Museum of Vertebrate Zoology, University of California, Berkeley, United States of America
}

Corresponding author: Laura Brenskelle (lbrensk@gmail.com)

Received: 08 Aug 2017 | Published: 09 Aug 2017

Citation: Brenskelle L, Wieczorek J, Guralnick R, Emery K, LeFebvre M (2017) Extending Darwin Core to incorporate data about material condition and absolute deep time. Proceedings of TDWG 1: e20126. https://doi.org/10.3897/tdwgproceedings.1.20126

\begin{abstract}
As part of efforts to mobilize zooarchaeological collections data, there is a strong need for new terms that can extend the Darwin Core standard in order to describe material condition, preparation history, and chronology. These data are important for understanding the full context of specimens from an array of natural and cultural heritage disciplines, especially those involving deep time, such as paleontology and zooarchaeology. These disciplines offer pre- and early Anthropocene biodiversity baselines to recognize and understand the deep history of human-environment interactions and use this information for research and conservation. They also provide an invaluable perspective about climate change in the past, thus providing insight into future climate change and impacts on ecology and biodiversity.
\end{abstract}

We propose two new extensions: one for chronology, and one for material condition. While Darwin Core does currently accommodate data about lithostratigraphy, the chronology extension will allow for sharing of absolute dates and dating protocols. Additionally, the material condition extension will provide proper means for sharing data that, thus far, have been lumped under the Darwin Core term 'preparations', which limits their discoverability to users. This includes data about skeletal elements, taphonomy, and preparation history. Here we present these two extensions to Darwin Core and open the discussion about improving the proposed terms and definitions in the extensions we developed. 


\section{Keywords}

Darwin Core, standards, discoverability, zooarchaeology, paleontology, absolute dates, chronology

\section{Presenting author}

Laura Brenskelle

\section{Funding program}

University of Florida Informatics Institute Seed Grant and University of Florida Research Opportunity Seed Fund. 